\title{
Low-beam-loss design of a compact, high-current deuteron radio frequency quadrupole accelerator
}

\author{
C. Zhang, ${ }^{1,2, *}$ Z. Y. Guo, ${ }^{1}$ A. Schempp, ${ }^{2}$ R. A. Jameson, ${ }^{2}$ J. E. Chen, ${ }^{1}$ and J. X. Fang ${ }^{1}$ \\ ${ }^{1}$ Key Laboratory of Heavy Ion Physics at Peking University, Ministry of Education, Beijing 100871, China \\ ${ }^{2}$ Institute for Applied Physics, Johann Wolfgang Goethe University, D-60054 Frankfurt am Main, Germany
} (Received 15 September 2004; published 26 October 2004)

A $201.5 \mathrm{MHz}, 50 \mathrm{~mA}, 2.0 \mathrm{MeV}$ deuteron radio frequency quadrupole accelerator is proposed as the neutron generator for the neutron experiment facility project at Peking University, China. Based on better understanding of beam losses, some new optimization procedures concerning both longitudinal and transverse dynamics are adopted. Accordingly, the beam transmission efficiency is improved from $91.2 \%$ to $98.3 \%$ and the electrode length is shortened from 2.91 to $2.71 \mathrm{~m}$. The fundamental physical analyses are performed to look inside the new design recipe and explain why it works.

DOI: 10.1103/PhysRevSTAB.7.100101

PACS numbers: 29.17.+w, 29.25.Dz, 29.27.-a

\section{INTRODUCTION}

For the Peking University (PKU) Neutron Experiment Facility, adequate neutron production could be achieved by using the ${ }^{9} \mathrm{Be}(d, n)$ reaction with a $50 \mathrm{~mA}$ peak current, $2.0 \mathrm{MeV}$ deuteron beam. A radio frequency quadrupole (RFQ)-based neutron source scheme is proposed due to its compact size and moderate investment [1]. The beam current and energy requirements are well within the capability of a RFQ [2], but severe economic and other constraints make the design problem unusually difficult.

The specifications and constraints of this project are as follows.

(a) A $50 \mathrm{~mA}$ peak current, $2.0 \mathrm{MeV}$ deuteron beam.

(b) As high as possible beam transmission efficiency, but for safety and ease of maintenance also emphasize that lost particles have as low as possible energy.

(c) Limited rf power source. A single rf transmitter will be available, using the THALES tetrode TH781 [3], which can deliver a maximum $400 \mathrm{~kW}$ peak power with $10 \%$ of duty factor and $1 \mathrm{~ms}$ of pulse duration at 201.5 MHz.

(d) The interelectrode voltage is constrained to $80 \mathrm{kV}$ and to be constant throughout the RFQ, which is convenient for tuning. At $201.5 \mathrm{MHz}$, this corresponds to a Kilpatrick factor of 1.8. This is a practical Kilpatrick factor commonly used to give the strongest possible focusing without having sparking.

(e) The deuteron injection energy to the RFQ is chosen as $0.05 \mathrm{MeV}$. A lower injection energy gives a shorter RFQ, minimizing construction costs and of power requirement, but making it more difficult to minimize beam losses. At $50 \mathrm{~mA}$ deuteron current, the spacecharge forces are high and a higher injection energy could be preferred, although at the expense of a longer RFQ and more rf power.

*Electronic address: zhang@iap.uni-frankfurt.de (f) Based on the survey on the specific shunt impedance of some existing four-rod RFQs, the kind of structure which will be used by the PKU deuteron RFQ for compact size [4], the total electrode length is expected to be less than $3 \mathrm{~m}$ with respect to the available rf power and the required beam current and energy.

(g) Lowest cost.

Table I then gives the basic parameters of the PKU deuteron RFQ.

These parameters resulted in the choice of a nontraditional beam dynamics design, as outlined below.

\section{BEAM DYNAMICS DESIGN AND OPTIMIZATION}

For RFQ beam dynamics design, the Los Alamos National Laboratory (LANL) Four-Section Procedure [5] is the traditional method, which divides an RFQ into four sequential sections. The radial matcher (RM) section serves to get high transverse capture at the entrance to the RFQ, the shaper (SH) and gentle buncher (GB) sections aim to longitudinally change the beam from continuous to well bunched. The real acceleration is achieved by the accelerator (ACC) section. The initial design of the PKU deuteron RFQ, which was optimized in a more traditional way, requires an RFQ length of $2.91 \mathrm{~m}$ and achieves a transmission of $91.2 \%$ (using 100000 macroparticles) [6]. The total amount of lost particles is still too high, which is undesirable from an

TABLE I. Basic parameters of the PKU deuteron RFQ.

\begin{tabular}{lc}
\hline \hline Frequency $[\mathrm{MHz}]$ & 201.5 \\
Input energy [MeV] & 0.05 \\
Output energy [MeV] & 2.00 \\
Peak beam current [mA] & 50 \\
Interelectrode voltage [kV] & 80 \\
Input emittance (Trans., $N, \mathrm{rms})[\pi \mathrm{mm} \mathrm{mrad}]$ & 0.2 \\
Duty factor [\%] & 10 \\
\hline \hline
\end{tabular}


induced radioactivity standpoint. In addition, the structure length is expected to be shortened a little, which is beneficial to leave more margin for the rf transmitter. Therefore, an improvement was sought.

Before optimization, it is very important to understand the beam loss well. In RFQ accelerators, there are two kinds of particle losses: $T$ type which is induced by transverse reasons and $\mathrm{L}$ type for longitudinal-induced one. L-type losses occur from beam bunching difficulties; T-type losses can occur when the beam is improperly injected into the RFQ (poor matching) or the spacecharge forces are not carefully enough controlled and particles hit the RFQ electrode surface. In fact, the Ltype particles are not really lost at once as they begin to be unstable. When some particles could not stay in the bucket of stable oscillations around the synchronous phase at a certain moment, normally they will slip toward the following bunch. Some of them could catch stable phases and get acceleration again, and others will get phase shifted; some may eventually be lost radially, otherwise they will go through the rest of the RFQ without or with some energy change. Obviously, L-type particles which could reach the RFQ exit are not so dangerous if the application of the RFQ does not strictly require very narrow energy spread. The PKU deuteron RFQ is such a case because it will be used for neutron production directly without any further acceleration structure.

The mechanisms of emittance growth, which are possible to lead to beam loss, have been extensively studied [7,8]. Briefly speaking, mismatching and resonances are two main reasons for instabilities. Especially the resonances, which could intrinsically redistribute the energy between the beam degrees of freedom with the cost of emittance growth, should be avoided. Resonances in RFQs could be distinguished into two classes: (1) the periodic-focusing-structure-driven resonance, which could be avoided by keeping the transverse zero-current phase advances of the particles' oscillating motion per focusing period $\sigma_{0 t}<90^{\circ}$; (2) the space-charge-driven resonances, which could be avoided by carefully choosing the parameters along the RFQ and let the trajectory of particles lie in the resonance-free areas of the Hofmann chart [9].

In a RFQ beam dynamics design, there are two very critical areas which directly affect the whole performance of the RFQ. The first one is the initial part of the RFQ including the RM section and the shaper, where the matching and shaping process have very important influences to the future beam motions. If they are not well done, a lot of particles will have great possibilities to be lost on the following way. To some extent, therefore, designing the initial part needs to be given more attention. The second "gate" for the RFQ design is the transition point between the GB section and the ACC section, which is very important for space-charge effects, because here the beam is maximally bunched but weakly accelerated. Therefore, the optimization design of the PKU deuteron RFQ was concentrated on these two areas and mainly achieved by the following three procedures.

The first optimization concept comes from the longitudinal point of view and mainly serves the first area. In the designing process of the initial scheme, the synchronous phase $\phi_{s}$ increases very fast in the SH and GB sections, e.g., $\phi_{s}$ has already been $-80^{\circ}$ at cell 50 , for shortening the total RFQ. This operation actually gets more acceleration in those two sections and meets the structure length demand of $<3 \mathrm{~m}$, but also violates the adiabatic bunching and results in a lot of L-type unstable particles, which could subsequently be lost. Figure 1 shows the variation of the synchronous phase throughout

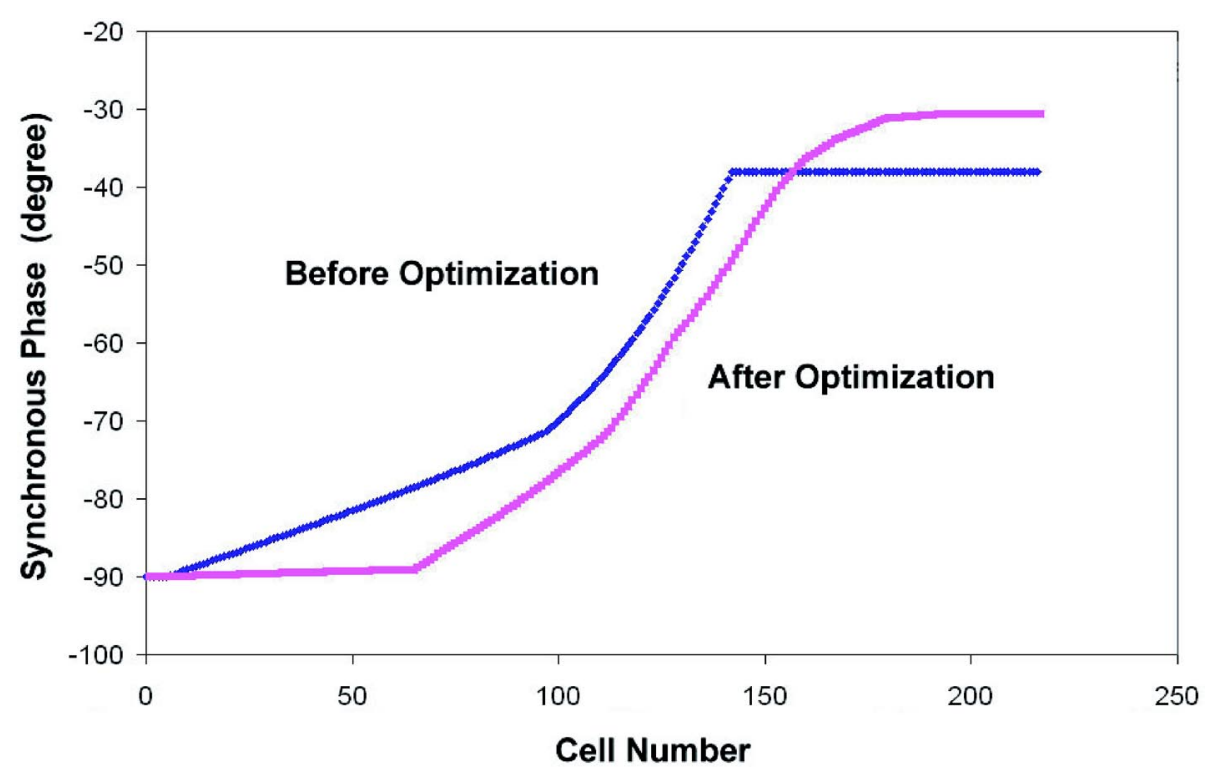

FIG. 1. (Color) Variation of the synchronous phase along the PKU deuteron RFQ. 
the RFQ before and after optimization. At the low energy end of the new design, $\phi_{s}$ is held at nearly $-90^{\circ}$ until cell 65 , which forms a good "porch" for the beam. Then it begins to increase a little fast, but before the end of gentle buncher it is still relatively smaller than the older design. Another difference is that $\phi_{s}$ is not kept constant in the ACC section like typical four-section designs but still gradually and slowly increasing, which is in favor of higher acceleration efficiency to counteract the length growth in the SH and GB sections for better longitudinal bunching.

The second optimization method is focused on the adjustment of the transverse focusing strength to improve the second gate. Before the ACC section, the space-charge forces of intense beams are increasing with decreasing beam bunch size at low velocities and behave most significantly at the end of the GB section. Then they will be reduced because of a rapidly increased acceleration rate. For a typical four-section design, the focusing parameter $B$ is held constant after the RM section in order to adapt the beam to a time-independent transverse focusing system. In fact, it is not necessary to hold $B$ fixed, and better to adapt it to the changing space-charge forces along the RFQ. Therefore, the method of adjusting the variation of the focusing parameter to keep a certain ratio between the focusing force and the space-charge force is employed for optimization. Figure 2 shows that $B$ has a nearly linear growth to the maximum value until the end of GB section and then decreases gradually to the exit of the RFQ. The decreasing focusing strength at the high energy end also has the merit of getting the chance to increase the electrode modulation $m$ a little, which is helpful to get higher accelerating gradient and shorten the total length.

Third, lots of simulation results show that in a traditional four-section design there are always two obvious peaks of beam losses located in the transition points of SH to GB and GB to ACC section, where beam parame- ters vary steeply. Consequently, a simple idea for optimization is to smooth most beam parameters' variation along the RFQ, especially at the last two critical points between sections. Then, different sections are gradually merged into together.

The beam dynamics design studies are done by the most classical reference software, the LANL RFQ design codes [10], and checked by the PTEQ code developed by Jameson, which is a very useful tool to look inside design schemes. Using 100000 macroparticles, the LANL codes give very good optimized results as expected. The beam transmission efficiency is increased from $91.2 \%$ to $98.3 \%$ and the electrode length is reduced from 2.91 to $2.71 \mathrm{~m}$. The simulation results of 10000 macro particles from PTEQ are also very similar, the beam transmission efficiency is improved from $91.0 \%$ to $99.2 \%$ and the length change is nearly the same.

From the LANL RFQ design codes, the main beam dynamics parameters of the PKU deuteron RFQ before and after optimization are listed in the Table II.

\section{COMPARISON AND ANALYSIS OF SCHEMES}

The equipartitioning procedure (EP), a design method very effective for high beam currents in the regime of high space-charge developed by Jameson [11], requires an energy balance between the transverse and longitudinal degrees of freedom along most of the RFQ. Figure 3 shows the typical picture of the parameter curves for checking the beam equipartitioning state, where $\varepsilon$ denotes the rms emittance, $\sigma$ the phase advance with beam current, sub- $t$ and sub- $l$ the transverse and longitudinal direction, sub- $n$ the normalized value, and $a$ and $b$ the transverse and longitudinal $\mathrm{rms}$ bunch radius. If the equipartitioning condition is satisfied, then $b / a=$ $e_{l n} / e_{t n}=\sigma_{t} / \sigma_{l}$ and $\left(e_{l n} * a\right) /\left(e_{t n} * b\right)=1$. The equipartitioning condition produces a good design by removing

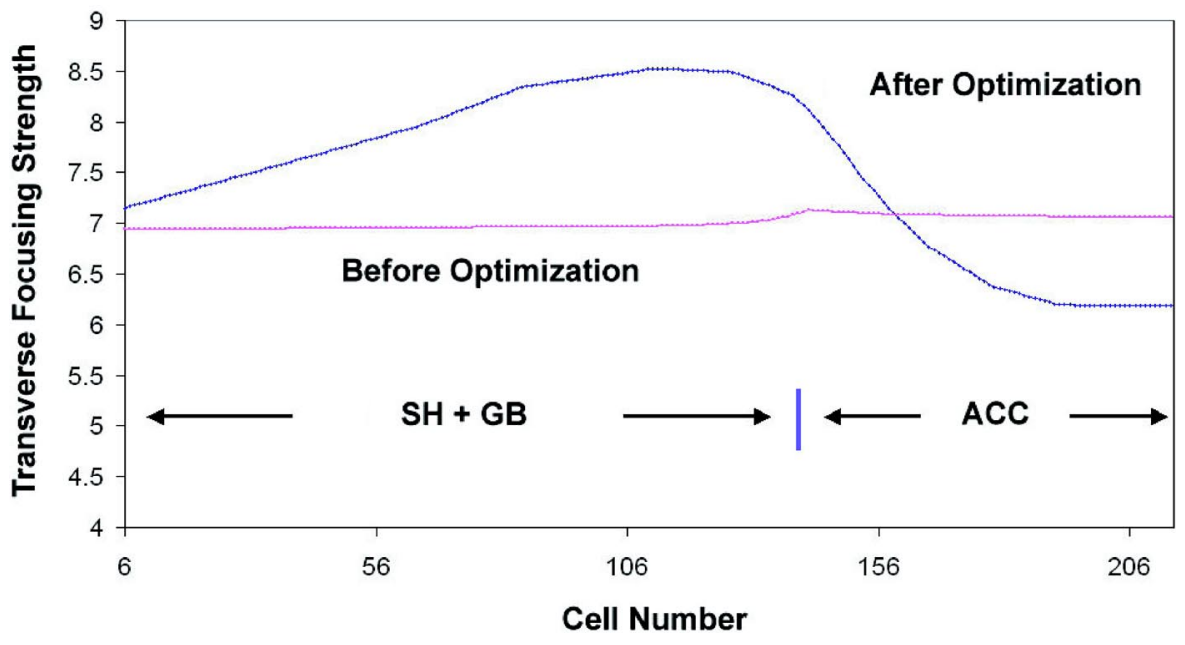

FIG. 2. (Color) Variation of the transverse focusing strength along the PKU deuteron RFQ. 
TABLE II. Main beam dynamics parameters of the PKU deuteron RFQ by LANL RFQ design codes.

\begin{tabular}{lcc}
\hline \multicolumn{1}{c}{ Parameters } & Before optimization & After optimization \\
\hline Synchronous phase $\left[{ }^{\circ}\right]$ & $-90--38.0$ & $-90--30.7$ \\
Electrode modulation & $1-1.73$ & $1-1.80$ \\
Output emittance $(N, \mathrm{rms}, x)[\pi \mathrm{mm} \mathrm{mrad}]$ & 0.2047 & 0.2079 \\
Output emittance $(N, \mathrm{rms}, y)[\pi \mathrm{mm}$ mrad] & 0.2087 & 0.2125 \\
Output emittance $(N, \mathrm{rms}, z)[\mathrm{MeV} \mathrm{deg}]$ & 0.9726 & 0.6249 \\
Minimum aperture $[\mathrm{cm}]$ & 0.262 & 0.267 \\
Electrode length $[\mathrm{m}]$ & 2.91 & 2.71 \\
Cell numbers & 216 & 216 \\
Beam transmission $[\%]$ & 91.2 & 98.3 \\
\hline \hline
\end{tabular}

free energy at the design tunes, which could feed emittance growth at resonances.

Using the EP parameters to check the PKU deuteron RFQ designs, Fig. 3 shows (1) a quasiequipartitioning state appears between the cell $50-150$ in the initial scheme and between the cell 100-120 in the new one; (2) except the quasiequipartitioning period, in the initial scheme all curves change much more steeply. Especially before optimization the $b / a$ value decreases very quickly within 10-20 cells at the very beginning of the RFQ, which results in an overbunched and mismatched beam with heavy beam losses and subsequent oscillations. Fortunately, because of the long quasiequipartitioning period the transmission efficiency of the old design could still be over $90 \%$. On the contrary, in the new design the $b / a$ curve goes down much more slowly and linearly to a relatively lower "balanced" value, which means better longitudinal bunching, and has a smaller oscillation.

In short, the conclusions of Fig. 3 are (1) the most important thing for a good design is that during the beam shaping and bunching the parameters' variations should be slow and linear enough, which is of great benefit to decrease the beam loss later in the RFQ; (2) the equipartitioning state is also very helpful espe- cially around the critical point, the end of the gentle buncher, but in the case of short-length-required RFQs strict equipartitioning is not necessary-it is more important to avoid parametric resonance areas as seen on the Hofmann chart.

Hofmann has introduced some useful charts to define thresholds for coherent instabilities in the particle distribution with several important quantities: the spacecharge-induced tune depressions, the ratio of emittances and phase advances between the longitudinal and transverse planes [12]. If a tune-depression point lies near the integer resonances where $\sigma_{l} / \sigma_{t}=1 / 2,1 / 3$, and $1 / 4$, the beam could be unstable and emittance growth will occur.

To investigate the trajectory of particles in the Hofmann chart more clearly, the parameter structures of the old design and the new one are divided into three and four parts, respectively, according to the characteristic of their zero-current phase advances' variation (the upper pictures in Fig. 4). The Hofmann charts of the two schemes are demonstrated in Fig. 4. They show the same beam traveling process as Fig. 3: first a bunching period to the end of the gentle buncher, then an oscillation period around the EP line, and finally an accelerating period, which departs from the EP condition.
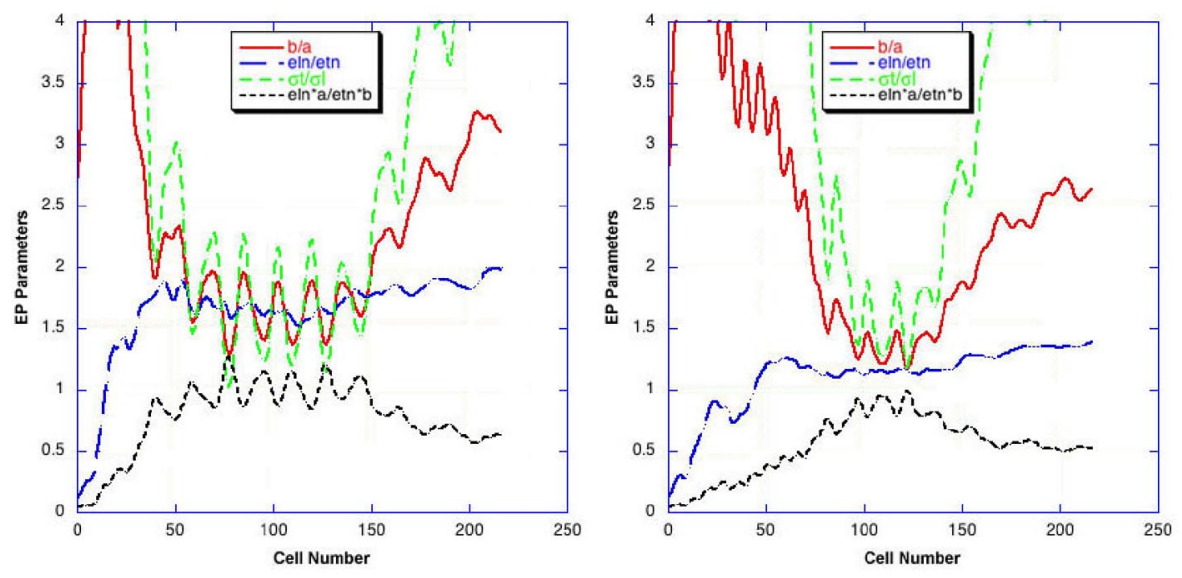

FIG. 3. (Color) EP parameters of the PKU deuteron RFQ (Left: before optimization; right: after optimization). 

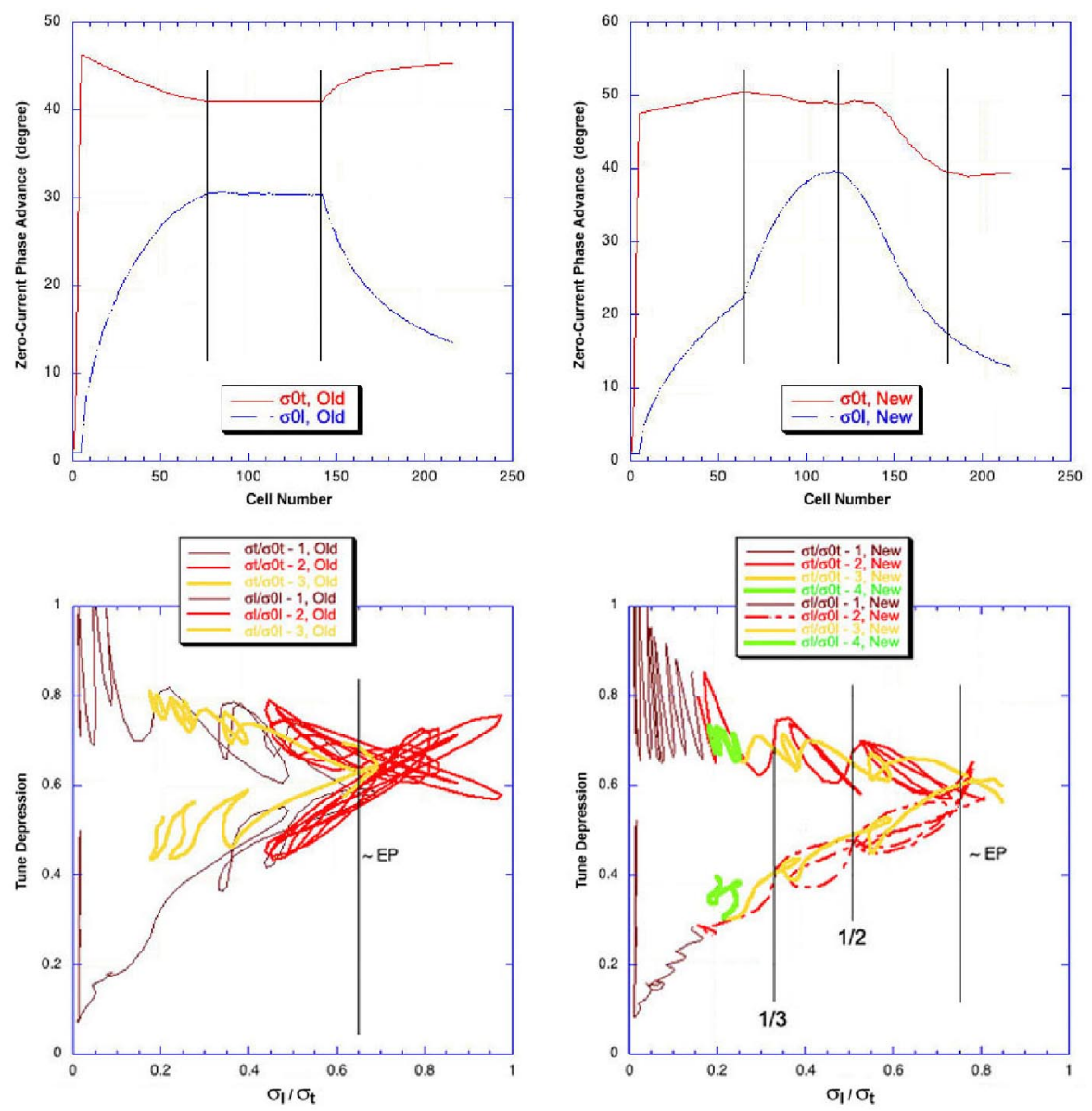

FIG. 4. (Color) Variation of the zero-current phase advances (upper) and Hofmann stability charts of the PKU deuteron RFQ (bottom) (left: before optimization; right: after optimization).

For both designs, the transverse tune depression $\sigma_{t} / \sigma_{0 t}$ at most positions of the RFQ is larger than 0.4 , a threshold where the space-charge and external focusing forces are approximately equal, except at the end of the third part and the whole fourth part of the beam trajectory in the new design. However, these are already very near the RFQ exit, so they do not have a very big influence except for some longitudinal emittance growth which is not very important for the PKU deuteron RFQ accelerator. Secondly,

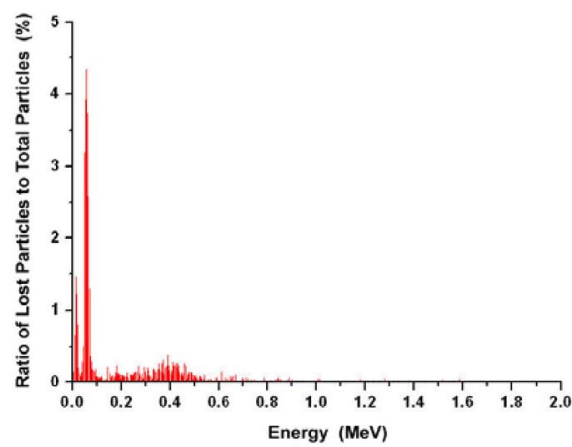

though there are several resonance peaks located in the beam trajectory, mostly the beam moves through them fairly fast. The beam trajectory oscillates around the " $1 / 2$ " resonance peak for a while in the old design, but is also nearly equipartitioned there so there is little free energy to feed the resonance. In the new design, the longitudinal trajectory encounters the " $1 / 3$ " and " $1 / 4$ " relatively weaker resonance peaks with tune depression below 0.4, and longitudinal emittance growth is seen.

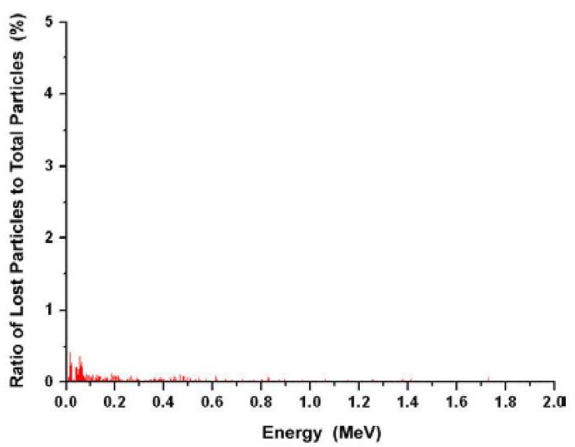

FIG. 5. (Color) Ratio of lost particles to total particles versus energy (left: before optimization; right: after optimization). 
From the pictures, the reason the beam transmission efficiency of these two non-EP designs are both over $90 \%$ could be easily understood, namely, that there are not very strong resonances. So, there are two ways to get stable designs: one is forcing the beam trajectory to stay in a resonance-free region, like the EP method, another is making it to move through resonance peaks quickly, like this PKU case.

As we discussed before, the particles lost at certain positions may not have the same energy as the synchronous particles there. Therefore, the energy spectrums of lost particles for both designs are plotted instead of showing the quantity of lost particles as a function of position along the RFQ. Compared with the initial design, it is obvious that in the new scheme the total amount of lost particles is reduced greatly. In both designs, most of the lost particles have relatively lower energy; only less than $0.2 \%$ of total particles are lost in the range of over $1.0 \mathrm{MeV}$ (Fig. 5). The old design has good performance at the high energy part of the RFQ, profiting from a longer quasiequipartitioning period and a larger final synchronous phase as $-38.0^{\circ}$. Though the new design uses $-30.7^{\circ}$, a relatively smaller synchronous phase at the ACC section with a few but acceptable beam loss here, it is worth it for getting short structure to save the limited rf power.

\section{CONCLUSIONS}

The RFQ design, which is framed by lots of boundary conditions such as the motivation of the project, the budget, and the available relative equipments, is both a practical problem and a physical one. Therefore, a final design scheme is normally a result of tradeoffs or a combination of various demands and different excellent design strategies. In the case of the PKU deuteron RFQ, very low beam loss is achieved by careful longitudinal bunching at the upstream of the RFQ and adjusting transverse focusing strength along the RFQ according to the variable space-charge effects. Though the equipartitioning procedure could put the beam trajectory in the resonance-free regions on the stability charts, it is just partly adopted by this design to meet the strict requirement of short length. The new design recipe successfully controls the instabilities in a compromising way, i.e., quickly passing through strong resonance peaks.

\section{ACKNOWLEDGMENTS}

C.Z. thanks the long-standing support and help of Gottlieb Daimler-Karl Benz Stiftung and Gesellschaft fuer Schwerionenforschung mbH (GSI), Germany. He also appreciates the kind initiatives of Professor H. Klein, the help of Dr. A. Bechtold, and the valuable discussion with Mr. G. Z. Jiang and Mr. Q. F. Luo on the development of RFQBAT code, a useful tool for the LANL RFQ design codes.

[1] Z.Y. Guo, C. Zhang, A. Schempp, J. E. Chen, J. X. Fang, Y. R. Lu, S. X. Peng, Z. Z. Song, J. X. Yu, and K. Zhu, in Proceedings of the 2004 Linac Conference, Lübeck, Germany (to be published).

[2] R. A. Jameson, R. Ferdinand, H. Klein, J. Rathke, J. Sredniawski, and M. Sugimoto, J. Nucl. Mater. (to be published).

[3] THALES Electron Devices Company, Neuilly-sur-Seine Cedex, France.

[4] A. Schempp, in Proceedings of the 1996 Linac Conference, Geneva, Switzerland, edited by C. Hill and M. Vretnar (CERN, Geneva, 1996), p. 53 (CD, ISBN ).

[5] K. R. Crandall, R. H. Stokes, and T. P. Wangler, in Proceedings of the 1979 Linac Conference, Montauk, NY (BNL Report No. BNL-51134, 1979), p. 205.

[6] C. Zhang, Z.Y. Guo, A. Schempp, J. E. Chen, and J. X. Fang, Nucl. Instrum. Methods Phys. Res., Sect. A 521, 326 (2004).

[7] I. Hofmann, in Applied Charged Particle Optics, Part C, edited by A. Septier (Academic, New York, 1983).

[8] T. P. Wangler, in High-Brightness Beams for Advanced Accelerator Applications, edited by WW. Destler and S. K. Guharay, AIP Conf. Proc. No. 253 (AIP, New York, 1991), p. 21.

[9] R. A. Jameson, in Advanced Accelerator Concepts, edited by J. S. Wirtele, AIP Conf. Proc. No. 279 (AIP, New York, 1993), p. 969.

[10] LANL Manual of RFQ Design Codes, Report No. LAUR-96-1836.

[11] R. A. Jameson, in Space Charge Dominated Beam Physics for Heavy Ion Fusion, edited by Y. K. Batygin, AIP Conf. Proc. No. 480 (AIP, New York, 1999), p. 21.

[12] I. Hofmann, in Proceedings of the Eighth European Particle Accelerator Conference, Paris, 2002 (EPSIGA and CERN, Geneva, 2002), p. 74. 